\title{
Geophysical modelling of 3D electromagnetic diffusion with multigrid
}

\author{
W. A. Mulder
}

Received: 19 December 2005 / Accepted: 8 May 2006 / Published online: 13 February 2007

(C) Shell International Exploration and Production B.V. 2007

\begin{abstract}
The performance of a multigrid solver for time-harmonic electromagnetic problems in geophysical settings was investigated. With the low frequencies used in geophysical surveys for deeper targets, the lightspeed waves in the earth can be neglected. Diffusion of induced currents is the dominant physical effect. The governing equations were discretised by the Finite-Integration Technique. The resulting set of discrete equation was solved by a multigrid method. The multigrid method provided excellent convergence with constant grid spacings, but not on stretched grids. The slower convergence rate of the multigrid method could be compensated by using bicgstab2, in which case multigrid acted as a preconditioner. Still, the overall performance was less than satisfactory with substantial grid stretching.
\end{abstract}

\section{Introduction}

The use of low-frequency electromagnetic signals for exploration was conceived by Conrad Schlumberger in 1912. He and his brother founded the company by that name. The technique was applied for mineral detection, for logging in boreholes [10,23], and for oil and

Communicated by C. Oosterlee.

W. A. Mulder $(\bowtie)$

Shell International Exploration and Production,

Kessler Park 1, 2288 GS Rijswijk, The Netherlands

e-mail: Wim.Mulder@shell.com

W. A. Mulder

Department of Geotechnology,

Faculty of Civil Engineering and Geosciences,

Delft University of Technology, Stevinweg 1,

2628 CN Delft, The Netherlands gas exploration from the surface [19,29,30,32]. Controlled-source electromagnetic (CSEM) measurements in marine environments were pioneered by the Scripps Institution of Oceanography $[8,9,36]$, and the Universities of Toronto [6,11] and Cambridge [20]. The technique was commercialised under the name seabed logging $[12,13]$. With the oil industry's move to increasingly deeper water, marine CSEM has gained some interest, in particular because in some cases the presence of hydrocarbon reservoirs could be related to anomalies that were extracted directly from data at a single frequency, without the need for inversion $[2,13]$.

In a typical experiment, currents are injected into the sea water by a wire that is towed by a ship. Detectors are placed at the sea bottom and record the horizontal components of the electric and magnetic fields. Resistive bodies can be detected by comparing data at large source-receiver distances to reference data, measured in a nearby area without hydrocarbon reservoirs or computed for an accurate background model without resistive bodies. The presence of oil and gas tends to increase the effective resistivity of the rock relative to formations that only contain some salty water in their pores. Modelling and inversion software is required for a more detailed interpretation of the data.

The propagation of electromagnetic waves in the earth can be described by Maxwell's equations combined with Ohm's law. At the low frequencies that are typically used, of the order of $1 \mathrm{~Hz}$, the light-speed waves have very small amplitudes and wavelengths that exceed the domain size. Diffusion is the dominant effect in the earth. This includes magneto-tellurics where the natural atmospheric background signals are used as well as controlled-source EM problems, but excludes ground penetrating radar applications. 
In two-dimensions, the problem can be reduced to a Poisson-type equation which can be solved either by a direct method using, among others, nested dissection [15], or iteratively by, for instance, the multigrid method. In three space dimensions, a direct solver may soon become too costly for large-scale problems and iterative methods are preferred.

The large null-space of the curl-curl operator that occurs in the equations may lead to problems. A system of Poisson-type equations can be obtained by a Helmholtz decomposition of the electric field into potentials $[4,5,17]$. This decomposition is unnecessary, because the problem of the null-space can be handled either explicitly by a divergence correction [18,28], or implicitly by solving small local systems [3]. The last approach is used here. The equations are discretised by the finiteintegration technique [7,33], which can be viewed as a finite-volume generalisation of Yee's scheme [35] for tensor-product Cartesian grids with variable grid spacings. The scheme is reviewed in Sect. 2.1. The multigrid solver is outlined in Sect. 2.2. It is a special case of the method presented in [14], but with a different restriction operator.

The multigrid method can be applied as a stand-alone solver or as a preconditioner for a Krylov subspace method. The latter may be necessary if the multigrid scheme has difficulties to remove certain type of errors. Here, we study convergence both for stand-alone multigrid and bicgstab2 $[16,31]$ preconditioned by multigrid.

A number of numerical tests are presented in Sect. 3. We start with an artificial, unphysical test problem based on sines and cosines. Next, a current source in a homogeneous formation and a realistic marine example are considered. Particular attention is paid to the effect of grid stretching.

A discussion of the numerical experiments and the main conclusions can be found in Sect. 4.

\section{Method}

\subsection{Discretisation}

The Maxwell equation for conducting media in the frequency domain at an angular frequency $\omega$ can be written as

$\iota \omega \mu_{0} \tilde{\sigma} \mathbf{E}-\nabla \times \mu_{r}^{-1} \nabla \times \mathbf{E}=-\imath \omega \mu_{0} \mathbf{J}_{S}$,

where the vector $\mathbf{E}(\omega, \mathbf{x})$ represents the electric field components as a function of angular frequency and position, and $\mathbf{J}_{S}(\omega, \mathbf{x})$ is a current source. Other quantities are $\tilde{\sigma}(\mathbf{x})=\sigma-\imath \omega \epsilon_{0} \epsilon_{r}$, with $\sigma(\mathbf{x})$ the conductivity, $\epsilon_{r}(\mathbf{x})$ the
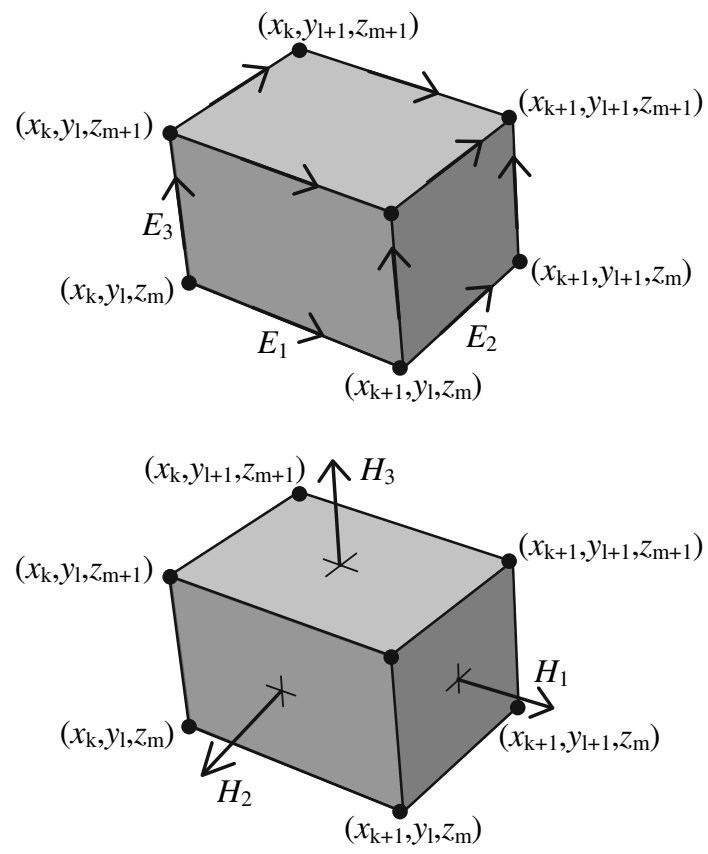

Fig. 1 The upper picture shows a grid cell with grid nodes and edge-averaged components of the electric field. The lower shows the magnetic field components that are obtained by taking the curl of the electric field

relative permittivity, $\mu_{r}(\mathbf{x})$ the relative permeability, and $\epsilon_{0}$ and $\mu_{0}$ their vacuum values. SI units will be used.

For geophysical measurements at frequencies around $1 \mathrm{~Hz}$, the term $\iota \omega \epsilon_{0} \epsilon_{r}$ in $\tilde{\sigma}$ can be neglected relative to $\sigma$, except in air. There, the wavelengths corresponding to this term exceed the earth's size. Therefore, the problem remains definite and multigrid may be expected to work. In the examples presented later, we will use $\epsilon_{r}=1$ in the geophysical examples, although $\epsilon_{r}=0$ will be considered in the artificial, unphysical test problems.

The equations are discretised by the finite-integration technique on a tensor-product cartesian grid allowing for grid stretching. The grid points define block-shaped cells. The electric field components are defined as edge averages. The magnetic field components $\mathbf{H}=(\nabla \times$ E) $/\left(\imath \omega \mu_{r} \mu_{0}\right)$ are normal to the faces of the cells, as sketched in Fig. 1. These components can be associated with edges of a dual grid with nodes at the barycentres of the original cells, drawn in Fig. 2. The curl of the magnetic field components can be associated with the edges of the original cells. The nodes of the original cells do not necessarily coincide with barycentres of the dual cells.

If the material properties are given as constant per cell, averaging is needed to obtain $\tilde{\sigma}$ at the edges where the electric field components reside. The area-averaged value over the face shared by the two dual cells that contain the edge of the original cell should be used [7]. For the implementation of the discretisation and the 


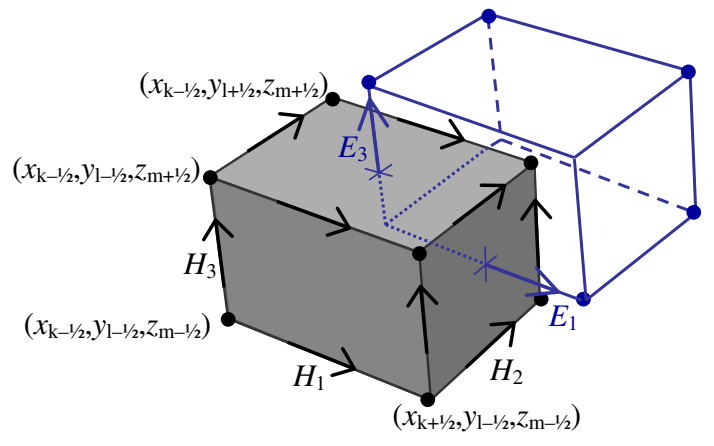

Fig. 2 The nodes of the dual grid are the barycentres of the original cells. A cell of the dual grid is shown in gray. The magnetic field components reside on the edges of the dual cells. Their curl can be associated with the edges of the original cells, where the electric field components live

multigrid solver, it was convenient to multiply the material properties by the cell volumes. This simplified the coarsening of material properties and residuals in the multigrid method.

To describe the averaging in more detail, we denote the nodes of the $N_{x} \times N_{y} \times N_{z}$ cells by $x_{k}\left(k=0, \ldots, N_{x}\right)$, $y_{l}\left(l=0, \ldots, N_{y}\right)$, and $z_{m}\left(m=0, \ldots, N_{z}\right)$. If

$V_{k+\frac{1}{2}, l+\frac{1}{2}, m+\frac{1}{2}}=\left(x_{k+1}-x_{k}\right)\left(y_{l+1}-y_{l}\right)\left(z_{m+1}-z_{m}\right)$,

is the volume of a cell, we define

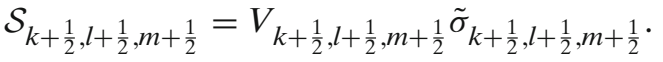

For the discretisation of, for instance, $\tilde{\sigma} E_{1}$ at the edge $\left(k+\frac{1}{2}, l, m\right)$, we use $\mathcal{S}_{k+\frac{1}{2}, l, m} E_{1, k+\frac{1}{2}, l, m} / V_{k+\frac{1}{2}, l, m}$ with

$$
\begin{aligned}
\mathcal{S}_{k+\frac{1}{2}, l, m}= & \frac{1}{4}\left(\mathcal{S}_{k+\frac{1}{2}, l-\frac{1}{2}, m-\frac{1}{2}}+\mathcal{S}_{k+\frac{1}{2}, l+\frac{1}{2}, m-\frac{1}{2}}\right. \\
& \left.+\mathcal{S}_{k+\frac{1}{2}, l-\frac{1}{2}, m+\frac{1}{2}}+\mathcal{S}_{k+\frac{1}{2}, l+\frac{1}{2}, m+\frac{1}{2}}\right),
\end{aligned}
$$

and

$V_{k+\frac{1}{2}, l, m}=\left(x_{k+1}-x_{k}\right) d_{l}^{y} d_{m}^{z}$.

Here $d_{k}^{x}=x_{k+\frac{1}{2}}-x_{k-\frac{1}{2}}, d_{l}^{y}=y_{l+\frac{1}{2}}-y_{l-\frac{1}{2}}$, and $d_{m}^{z}=$ $z_{m+\frac{1}{2}}-z_{m-\frac{1}{2}}$ are the lengths of the edges of the dual cells. Note that in the interior, $V_{k+\frac{1}{2}, l, m}$ is the intersection of the two dual cells containing edge $\left(k+\frac{1}{2}, l, m\right)$ with the four cells surrounding that edge, as sketched in Fig. 3. In the implementation, it was convenient to work with $\mathcal{S}_{k+\frac{1}{2}, l, m} E_{1, k+\frac{1}{2}, l, m}$, meaning that the residual for the first component was multiplied by $V_{k+\frac{1}{2}, l, m}$. We will call this volume the dual edge-volume of that edge. The other two components are dealt with in a similar way.

To obtain the magnetic field components from the curl of the electric field, we need $1 / \mu_{r}$ at the faces of

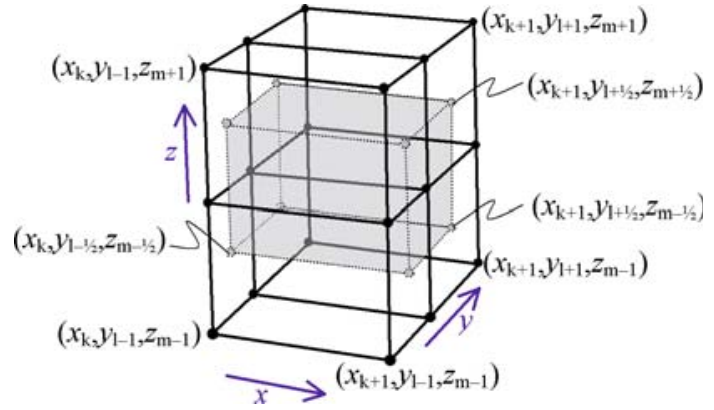

Fig. 3 The conductivity should be averaged over the area of a face of the dual grid. For material properties that are piecewise constant per cell, the same result can be obtained by volumeweighted averaging. The volume over which the averaging should be carried out for a component of the electric field is the intersection of the two dual cells containing the edge, with the four cells surrounding that edge. Here, four of the six faces bounding this dual edge-volume for the first electric field component are sketched

the original cells. This requires averaging of $1 / \mu_{r}$ over an edge of the the dual cells [27]. For values that are piecewise constant per cell, this is the same as volumeweighted averaging of the values of $1 / \mu_{r}$ from the two cells adjacent the face where the curl of the electric field lives. The volume-weighted averaging is based on the volumes of the two neighbouring cells.

We have assumed that the material properties are given as averages per cell. A more accurate approach incorporates variations of the material inside the cell into the averaging procedure [7]. That approach was not considered here.

The components of the current source $\mathbf{J}_{S}$ can be associated with the edges in the same way as the electric field components. The components represent averages over the faces of the dual cells, the same as involved in the curl of the magnetic field components. If the integral of a component of $\mathbf{J}_{s}$ over the dual face is also multiplied by the length of the edge on which it lives, we obtain a discretisation of the current-source component times the dual edge-volume.

With this discretisation, the solution has second-order accuracy for constant coefficients [22]. This also holds when the coefficients vary across the domain, except for a discontinuous $\mu_{r}$ which, in general, leads to a firstorder error in the solution [27].

Perfectly electric conducting boundary conditions were used for the numerical experiments in this paper.

\subsection{Multigrid}

The multigrid method resembles the one described by Feigh et al. [14], but here the natural coarsening of eight fine-grid cells contained in a single coarse-grid cell will 
be used. The material properties multiplied by their cell volumes are just added for restriction and used for a coarse-grid operator that is based on the same discretisation as the fine-grid operator. For the restriction of the residual, two restriction operators were initially considered. The first amounts to integrating over all the dual edge-volumes contained in the coarse-grid volume of a given edge, with suitable scaling proportional to the fractions of the fine-grid dual edge-volumes contained inside the coarse-grid dual edge-volumes. This reduces to full weighting if the cells have fixed widths.

To explain in this in some detail, we consider the simple case of $N_{x} \times N_{y} \times N_{z}$ cells with $N_{x}=N_{y}=$ $N_{z}=2^{M}$ and integer $M \geq 1$. A coarse grid is defined by selecting every other point of $x_{k}\left(k=0, \ldots, N_{x}\right)$, $y_{l}\left(l=0, \ldots, N_{y}\right)$, and $z_{m}\left(m=0, \ldots, N_{z}\right)$. The coarsegrid nodes become $\left(x_{2 K}, y_{2 L}, z_{2 M}\right)$ with $K=0, \ldots, N_{x} / 2$, $L=0, \ldots, N_{y} / 2$, and $M=0, \ldots, N_{z} / 2$. To describe the restriction operator, we only look at the first component of the residual $\mathbf{r}$. As mentioned before, the residuals were multiplied by the dual edge-volumes. The components of the residual reside on the same edges as the components of the electric field. The first coarse-grid component is $r_{1, K+\frac{1}{2}, L, M}^{2 h}$, where the superscript $2 h$ is used to denote the coarse-grid values. Let the index $K$ on the coarser grid correspond to $k=2 K$ on the fine grid. Likewise, $l=2 L$ and $m=2 M$ on the fine grid. The restriction of this component is given by

$$
\begin{aligned}
r_{1, K+\frac{1}{2}, L, M}^{2 h}= & \sum_{j_{2}=-1}^{1} \sum_{j_{3}=-1}^{1} w_{L, j_{2}}^{y} w_{M, j_{3}}^{z} \\
& \times\left(r_{1, k+\frac{1}{2}, l+j_{2}, m+j_{3}}^{h}+r_{1, k+\frac{3}{2}, l+j_{2}, m+j_{3}}^{h}\right),
\end{aligned}
$$

where

$$
\begin{aligned}
w_{L,-1}^{y} & =\left(y_{l-\frac{1}{2}}^{h}-y_{L-\frac{1}{2}}^{2 h}\right) / d_{l-1}^{y}, \\
w_{L, 0}^{y} & =1, \\
w_{L, 1}^{y} & =\left(y_{L+\frac{1}{2}}^{2 h}-y_{l+\frac{1}{2}}^{h}\right) / d_{l+1}^{y},
\end{aligned}
$$

and

$$
\begin{aligned}
w_{M,-1}^{z} & =\left(z_{m-\frac{1}{2}}^{h}-z_{M-\frac{1}{2}}^{2 h}\right) / d_{m-1}^{z}, \\
w_{M, 0}^{z} & =1 \\
w_{M, 1}^{z} & =\left(z_{M+\frac{1}{2}}^{2 h}-z_{m+\frac{1}{2}}^{h}\right) / d_{m+1}^{z}
\end{aligned}
$$

The restriction operators for the other components of the residual follow in a similar way.

The second, simpler restriction operator agrees with [14] and amounts to an addition of the fine-grid residuals residing on a coarse-grid edge, with a suitable scaling to account for the dual edge-volume. For the first component of the residual, we have

$r_{1, K+\frac{1}{2}, L, M}^{2 h}=w_{L}^{y} w_{M}^{z}\left(r_{1, k+\frac{1}{2}, l, m}^{h}+r_{1, k+\frac{3}{2}, l, m}^{h}\right)$,

with $w_{L}^{y}=\left(y_{L+\frac{1}{2}}^{2 h}-y_{L-\frac{1}{2}}^{2 h}\right) / d_{l}^{y}$, and $w_{M}^{z}=\left(z_{M+\frac{1}{2}}^{2 h}-\right.$ $\left.z_{M-\frac{1}{2}}^{2 h}\right) / d_{m}^{z}$. Numerical experiments on stretched grids showed that this restriction operator led to divergence in some cases. The examples presented in the next section were all computed with the other restriction operator.

For the prolongation of the coarse-grid corrections to the electric field components, piecewise constant interpolation was used in the direction of the component, and linear and bilinear interpolation in the plane perpendicular to the component. This is the same as the adjoint of Eq. (2).

Symmetric block Gauss-Seidel (SBGS) [3] in lexicographical ordering was applied as a smoother in F-cycles with two post-smoothing sweeps, being one SBGS relaxation step. In the examples where multigrid acts as a preconditioner, a single F-cycle was performed.

\section{Examples}

\subsection{Artificial test problem}

A test problem based on eigenfunctions can be found in [4]. Here, this problem is modified to allow for the use of perfectly electric conducting (PEC) boundary conditions. The domain is $\Omega=[0,2 \pi]^{3} \mathrm{~m}^{3}$. Define $\psi=$ $\sin k x \sin l y \sin m y$, with $k, l$, and $m$ positive integers. Let the exact solution be

$E_{1}=a_{1} \partial_{x} \psi, \quad E_{2}=a_{2} \partial_{y} \psi, \quad E_{3}=a_{3} \partial_{z} \psi$.

The domain $\Omega$ is split into parts $\Omega_{1}$ with $z<\pi$ and $\Omega_{2}$ for $z>\pi$, so that $\Omega$ is the union of their closure. The conductivity $\sigma=\sigma_{0}+\sigma_{1}(x+1)(y+2)(z-\pi)^{2}$ in $\Omega_{1}$, and $\sigma=\sigma_{0}$ in $\Omega_{2}$. We set $\epsilon_{r}=0$, which is unphysical, $\mu_{r}=1$, and $\omega=10^{6} \mathrm{~Hz}$. The other parameters are chosen to be $a_{1}=a_{2}=-2 \mathrm{~V}, a_{3}=1 \mathrm{~V}, k=l=m=1, \sigma_{0}=10 \mathrm{~S} / \mathrm{m}$, and $\sigma_{1}=1 \mathrm{~S} / \mathrm{m}$. Note that $\psi$ is based on sine functions, causing the tangential components of the electric field to vanish at the boundaries in agreement with the PEC boundary conditions. The current source is defined by $\mathbf{J}_{s}=-\tilde{\sigma} \mathbf{E}+\nabla \times(\iota \omega \mu)^{-1} \nabla \times \mathbf{E}$, using the exact solution. This results in 
Table 1 Number of iterations and solution errors for the first test problem with $\sigma_{0}=10 \mathrm{~S} / \mathrm{m}$ and $\sigma_{1}=1 \mathrm{~S} / \mathrm{m}$

\begin{tabular}{rlllll}
\hline$N$ & $h_{\max }$ & MG & bi & $\epsilon_{2} / h_{\max }^{2}$ & $\epsilon_{\max } / h_{\max }^{2}$ \\
\hline 16 & 0.39 & 8 & 6 & $8.6 \times 10^{-2}$ & 0.21 \\
32 & 0.20 & 8 & 7 & $8.8 \times 10^{-2}$ & 0.24 \\
64 & 0.098 & 8 & 7 & $8.9 \times 10^{-2}$ & 0.24 \\
128 & 0.049 & 8 & 6 & $8.9 \times 10^{-2}$ & 0.24 \\
\hline
\end{tabular}

$$
\begin{aligned}
\mathbf{J}_{s}= & -\tilde{\sigma}\left(\begin{array}{l}
a_{1} \partial_{x} \psi \\
a_{2} \partial_{y} \psi \\
a_{3} \partial_{z} \psi
\end{array}\right) \\
& +(l \omega \mu)^{-1}\left(\begin{array}{l}
{\left[l^{2}\left(a_{1}-a_{2}\right)+m^{2}\left(a_{1}-a_{3}\right)\right] \partial_{x} \psi} \\
{\left[k^{2}\left(a_{2}-a_{1}\right)+m^{2}\left(a_{2}-a_{3}\right)\right] \partial_{y} \psi} \\
{\left[k^{2}\left(a_{3}-a_{1}\right)+l^{2}\left(a_{3}-a_{2}\right)\right] \partial_{z} \psi}
\end{array}\right),
\end{aligned}
$$

The tests were carried out with $\sigma_{0}=10 \mathrm{~S} / \mathrm{m}$ and $\sigma_{1}=1 \mathrm{~S} / \mathrm{m}$. Table 1 lists the number of iterations and errors. The number of cells in each coordinate direction is given by $N_{x}=N_{y}=N_{z}=N$. For each grid, the number of iterations with stand-alone multigrid (MG) and with multigrid as preconditioner for bicgstab2 (bi) is given. Note that bicgstab2 costs a bit more per iteration because it requires an additional evaluation of the residual. Also, each bicgstab2 step is counted as two iterations because it involves two multigrid cycles. Because convergence checks are carried out halfway and at the end of a full iteration step, the method may stop after on odd number of iterations. The iterations were stopped when the $\ell_{2}$-norm of residual had dropped by a factor $10^{-8}$ from its original value for a zero solution.

The error in the numerical solution is listen in the 2-norm and maximum norm. If the grid has $N_{x} \times N_{y} \times N_{z}$ cells with nodes $\left(x_{k}, y_{l}, z_{m}\right)$, where $k=0, \ldots, N_{x}, l=$ $0, \ldots, N_{y}$, and $m=0, \ldots, N_{z}$, the 2-norm of the first electric-field component can be defined as

$\left\|E_{1}\right\|_{2}=\left[\sum_{k=0}^{N_{x}-1} \sum_{l=0}^{N_{y}} \sum_{m=0}^{N_{z}}\left|E_{1, k+\frac{1}{2}, l, m}\right|^{2} V_{k+\frac{1}{2}, l, m}\right]^{1 / 2}$,

and similarly for the other components. The solution error is

$\ell_{2}=\left[\sum_{n=1}^{3}\left\|E_{n}-E_{n}^{\text {exact }}\right\|_{2}^{2}\right]^{1 / 2}$,

and the relative error

$\epsilon_{2}=\ell_{2}\left[\sum_{n=1}^{3}\left\|E_{n}^{\text {exact }}\right\|_{2}^{2}\right]^{-1 / 2}$.
Table 2 Number of iterations and solution errors for the first test problem with $\sigma_{0}=10 \mathrm{~S} / \mathrm{m}, \sigma_{1}=1 \mathrm{~S} / \mathrm{m}$, and power-law grid stretching with $\alpha=0.04$

\begin{tabular}{rrrrll}
\hline$N$ & $h_{\max }$ & MG & bi & $\epsilon_{2} / h_{\max }^{2}$ & $\epsilon_{\max } / h_{\max }^{2}$ \\
\hline 16 & 0.45 & 9 & 7 & $8.2 \times 10^{-2}$ & 0.18 \\
32 & 0.26 & 11 & 8 & $8.0 \times 10^{-2}$ & 0.16 \\
64 & 0.17 & 12 & 14 & $7.4 \times 10^{-2}$ & 0.15 \\
128 & 0.13 & 83 & 33 & $6.9 \times 10^{-2}$ & 0.14 \\
\hline
\end{tabular}

For the maximum norm, we have

$$
\left\|E_{1}\right\|_{\max }=\max _{k=0, \ldots, N_{x}-1} \max _{l=0, \ldots, N_{y}} \max _{m=0, \ldots, N_{z}}\left|E_{1, k+\frac{1}{2}, l, m}\right|,
$$

and similarly for the other components. The maximum error is denoted by

$$
\ell_{\max }=\max _{n=1,2,3}\left\|E_{n}-E_{n}^{\text {exact }}\right\|_{\max }
$$

and the corresponding relative error by

$\epsilon_{\max }=\ell_{\max }\left[\max _{n=1,2,3}\left\|E_{n}^{\text {exact }}\right\|_{\max }\right]^{-1}$.

For the exact solution, the point-values at the edge-midpoints were used.

The results in the Table 1 show grid-independent convergence for the multigrid method. The number of iterations with bicgstab2 is smaller, but this is hardly worth the extra cost in terms of cpu-time and storage. The six bicgstab2 iterations for the grid with $128^{3}$ cells, for instance, took only $10 \%$ less cpu time than the eight iterations with stand-alone multigrid. The errors confirm the second-order accuracy of the solution. The errors were divided by the square of the largest cell width $h_{\max }$, which is the maximum value over all the cell widths in the three coordinate directions.

Next, the effect of grid stretching was investigated. The grid stretching is carried out in such a way that the ratio between neighbouring cell widths in each coordinate is $1+\alpha$ when marching away from the origin (see power-law stretching in Appendix). An equidistant grid is obtained for $\alpha=0$. Results for grid stretching with $\alpha=0.04$ are listed in Table 2. Apparently, the grid-independent convergence rates of the multigrid scheme are lost. The bicgstab2 method now pays off, as it is able to deal with the slowly converging components of the solution. The errors measured by $\epsilon_{2}$ and $\epsilon_{\max }$ agree with the expected second-order accuracy of the solution.

We conclude that grid stretching does not do a whole lot of good for the current problem in terms of convergence speed. 


\subsection{Variable permittivity}

The same domain is considered as before, with conductivity $\sigma=1 \mathrm{~S} / \mathrm{m}$ and the unphysical choice of $\epsilon_{r}=0$. The permittivity is piecewise constant with $\mu_{r}(z)=\mu_{r}^{L}$ for $z<\pi$ and $\mu_{r}(z)=\mu_{r}^{R}$ for $z>\pi$. The source terms are set to

$J_{1}=\quad \cos (x) \sin (y) \sin (z)\left[1-3 /\left(\imath \omega \mu_{0} \mu_{r}(z)\right)\right]$,

$J_{2}=\sin (x) \cos (y) \sin (z)\left[1-3 /\left(\imath \omega \mu_{0} \mu_{r}(z)\right)\right]$,

$J_{3}=-2 \sin (x) \sin (y) \cos (z)\left[1-3 /\left(\imath \omega \mu_{0} \mu_{r}(z)\right)\right]$,

The solution is

$E_{1}=-\cos (x) \sin (y)\left[\sin (z)+c g_{1}(z)\right]$,

$E_{2}=-\sin (x) \cos (y)\left[\sin (z)+c g_{1}(z)\right]$,

$E_{3}=2 \sin (x) \sin (y)\left[\cos (z)+c g_{3}(z)\right]$.

Let $q^{L, R}=\sqrt{2-\imath \omega \mu_{0} \mu_{r}^{L, R}}$. Then

$g_{1}^{L}(z)=2 \sinh \left(q^{L} z\right)$,

$g_{3}^{L}(z)=-2 \cosh \left(q^{L} z\right) / q^{L}$,

for $z<\pi$, whereas for $z>\pi$,

$g_{1}^{R}(z)=2 a \sinh \left(q^{R}(2 \pi-z)\right)$,

$g_{3}^{R}(z)=2 a \cosh \left(q^{R}(2 \pi-z)\right) / q^{R}$.

Here $a=\sinh \left(q^{L} \pi\right) / \sinh \left(q^{R} \pi\right)$ and

$c=\frac{3}{2 \imath \omega \mu_{0}}\left(\frac{1}{\mu_{r}^{R}}-\frac{1}{\mu_{r}^{L}}\right) /\left\{\frac{\cosh \left(q^{L} \pi\right)}{q^{L}}+a \frac{\cosh \left(q^{R} \pi\right)}{q^{R}}\right\}$.

Note that $g_{1}(z)$ is continuous across $z=\pi$, with a discontinuous derivative, and $g_{3}(z)$ is discontinuous across $z=\pi$.

We ran tests with $\mu_{r}^{L}=1$ and $\mu_{r}^{R}=2$. Convergence results are listed in Tables 3 and 4. Again, convergence is slower on stretched grids. The errors suggest that the solution has second-order accuracy in this example.

\subsection{Homogeneous problem with current source}

An example that is slightly more realistic from a geophysical point of view, is a point current source in a homogeneous formation. We choose the following

Table 3 Number of iterations and solution errors for the problem with constant $\sigma$ and variable $\mu_{r}$, without grid stretching

\begin{tabular}{rlrlll}
\hline$N$ & $h_{\max }$ & MG & bi & $\epsilon_{2} / h_{\max }^{2}$ & $\epsilon_{\max } / h_{\max }^{2}$ \\
\hline 16 & 0.39 & 11 & 8 & 0.13 & 0.19 \\
32 & 0.20 & 10 & 7 & 0.13 & 0.22 \\
64 & 0.098 & 9 & 7 & 0.13 & 0.24 \\
128 & 0.049 & 9 & 7 & 0.13 & 0.25 \\
\hline
\end{tabular}

Table 4 Number of iterations and solution errors for the problem with constant $\sigma$ and variable $\mu_{r}$, using grid stretching with $\alpha=0.04$

\begin{tabular}{rrrrll}
\hline$N$ & $h_{\max }$ & MG & bi & $\epsilon_{2} / h_{\max }^{2}$ & $\epsilon_{\max } / h_{\max }^{2}$ \\
\hline 16 & 0.45 & 12 & 9 & $8.6 \times 10^{-2}$ & $1.3 \times 10^{-1}$ \\
32 & 0.26 & 14 & 10 & $6.3 \times 10^{-2}$ & $1.0 \times 10^{-1}$ \\
64 & 0.17 & 30 & 16 & $5.1 \times 10^{-2}$ & $6.0 \times 10^{-2}$ \\
128 & 0.13 & 177 & 38 & $5.0 \times 10^{-2}$ & $5.7 \times 10^{-2}$ \\
\hline
\end{tabular}

Table 5 Number of iterations and solution errors in a subset of the domain for the homogeneous test problem with a current point source and no stretching

\begin{tabular}{rrllll}
\hline$N$ & $h_{\max }$ & MG & bi & $\epsilon_{2} / h_{\max }^{2}$ & $\epsilon_{\max } / h_{\max }^{2}$ \\
\hline 16 & 125 & 10 & 8 & $2.4 \times 10^{-5}$ & $4.1 \times 10^{-5}$ \\
32 & 63 & 13 & 9 & $2.7 \times 10^{-5}$ & $7.1 \times 10^{-5}$ \\
64 & 31 & 13 & 9 & $2.4 \times 10^{-5}$ & $6.8 \times 10^{-5}$ \\
128 & 16 & 13 & 9 & $2.8 \times 10^{-5}$ & $7.2 \times 10^{-5}$ \\
\hline
\end{tabular}

parameters: $\omega / 2 \pi=10 \mathrm{~Hz}, \sigma=1 \mathrm{~S} / \mathrm{m}, \mu_{r}=1$, and $\epsilon_{r}=$ 1. The domain is the cube $[-1,1] \times[-1,1] \times[-1,1] \mathrm{km}^{3}$. The current source $\mathbf{J}_{s}=\left(\begin{array}{lll}0 & 0 & 1\end{array}\right)^{\top} \delta(\mathbf{x}) \mathrm{A} / \mathrm{m}^{2}$ is placed at the origin.

The problem is discretised on an equidistant grid with $N_{x}=N_{y}=N_{z}=N$. Also, power-law grid stretching (Appendix) is considered with cell widths increasing away from the source. For the discrete source representation, the adjoint of trilinear interpolation is used. The iterations are stopped if the $\ell_{2}$-norm of the residual has dropped by a factor $10^{-8}$ from its initial value for a zero solution.

The exact solution can be found in [32]. The PEC boundary conditions do not agree with this solution, but still can be used if they are placed sufficiently far away from the source. Because the exact solution has a $r^{-3}$ singularity at the source, we will not observe a decrease of the error under grid refinement. The point current source is effectively represented as finite-length source, with a length that depends on the edge or edges on which the source lives. In the present example, the source position coincides with a node and is represented by contributions on the edges just above and below that node. Changing the length of these edges will strongly affect the near field, resulting in a loss of second-order accuracy. The far field will not depend of the details of the source, only on its properties in an average sense. By measuring the error only outside the central cube $[-250,250]^{3} \mathrm{~m}^{3}$, the results shown in Tables 5,6 , and 7 were obtained. Excellent iteration counts are observed without grid stretching. If the amount of stretching is increased above a few percent, convergence rates deteriorate. 
Table 6 Number of iterations and solution errors in a subset of the domain for the homogeneous test problem with a current point source and power-law grid stretching with $\alpha=0.02$

\begin{tabular}{rrcccc}
\hline$N$ & $h_{\max }$ & MG & bi & $\epsilon_{2} / h_{\max }^{2}$ & $\epsilon_{\max } / h_{\max }^{2}$ \\
\hline 16 & 134 & 11 & 8 & $2.0 \times 10^{-5}$ & $7.0 \times 10^{-5}$ \\
32 & 72 & 13 & 9 & $1.6 \times 10^{-5}$ & $8.0 \times 10^{-5}$ \\
64 & 42 & 13 & 9 & $7.9 \times 10^{-6}$ & $2.9 \times 10^{-5}$ \\
128 & 27 & 13 & 9 & $6.2 \times 10^{-6}$ & $7.4 \times 10^{-6}$ \\
\hline
\end{tabular}

Table 7 Number of iterations and solution errors in a subset of the domain for the homogeneous test problem with a current point source and power-law grid stretching with $\alpha=0.05$

\begin{tabular}{rrrrll}
\hline$N$ & $h_{\max }$ & MG & bi & $\epsilon_{2} / h_{\max }^{2}$ & $\epsilon_{\max } / h_{\max }^{2}$ \\
\hline 16 & 147 & 11 & 8 & $1.6 \times 10^{-5}$ & $5.4 \times 10^{-5}$ \\
32 & 88 & 14 & 10 & $6.5 \times 10^{-6}$ & $2.9 \times 10^{-5}$ \\
64 & 60 & 26 & 14 & $2.2 \times 10^{-6}$ & $4.0 \times 10^{-6}$ \\
128 & 50 & 185 & 47 & $1.9 \times 10^{-6}$ & $8.9 \times 10^{-7}$ \\
\hline
\end{tabular}

\subsection{Marine example}

A more realistic subsurface model was constructed by modifying the SEG/EAGE salt model [1]. This model was designed for simulating seismic wave propagation and contains a complex salt body surrounded by sediments. The sea water has depths around $120 \mathrm{~m}$. Its dimensions are $13,500 \times 13,480 \times 4,680 \mathrm{~m}$. Because CSEM requires a deeper sea than provided by this model, the water depth was increased with $500 \mathrm{~m}$ by moving down the model. Next, velocities were replaced by conductivities. The water velocity of $1,500 \mathrm{~m} / \mathrm{s}$ was replaced by $1 / 0.3 \mathrm{~S} / \mathrm{m}$. Velocities above $4,000 \mathrm{~m} / \mathrm{s}$, indicative of salt, were replaced by $1 / 30 \mathrm{~S} / \mathrm{m}$. Below 3,956 m, basement was set to $500 \mathrm{~S} / \mathrm{m}$. The conductivity of the sediments was determined by $\sigma_{\text {sed }}=(v / 1700)^{-3.88} \mathrm{~S} / \mathrm{m}$, with the velocity $v$ in $\mathrm{m} / \mathrm{s}$. This choice was motivated by [21]. For air, $\sigma$ was set to $10^{-10} \mathrm{~S} / \mathrm{m}$. The conductivity was mapped to a new grid with hyperbolic cosine stretching. The horizontal dimensions were the same as for the original model, whereas $z$ ranged from $-5,000$ to $5,000 \mathrm{~m}$. A total of 31 receivers were positioned at the sea-bottom between $x=4,000$ and $10,000 \mathrm{~m}$ at $200 \mathrm{~m}$ spacing and with $y=0 \mathrm{~m}$. Depths ranged between 605 and $625 \mathrm{~m}$. A subset of the model is displayed in Fig. 4. For its discrete representation, the exact cell averages are computed. The frequency is $\omega / 2 \pi=5 \mathrm{~Hz}$. A finite-length current source with normalised strength was placed on the interval between $(x, y, z)=(6,400,6,500,500)$ and $(x, y, z)=(6,600,6,500,500)$, all in meters.

Convergence results for a grid with $128^{3}$ cells are listed in Table 8 . The grid stretching is controlled by the hyperbolic cosine function (Appendix), using a smallest

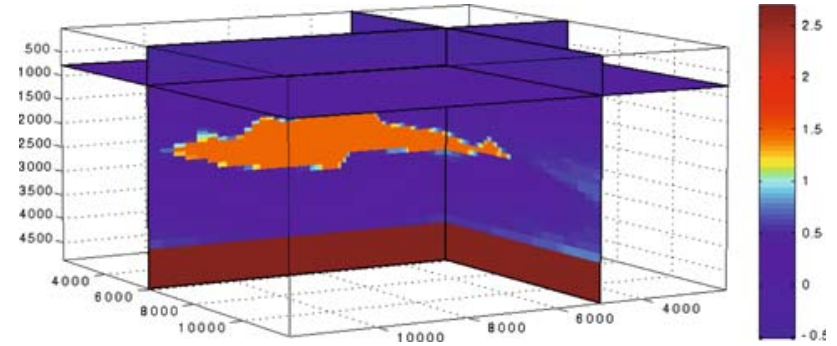

Fig. 4 Model derived from the SEG/EAGE salt model. Shown is the base 10 logarithm of the resistivity in $\Omega \mathrm{m}$ for a subset of the model

Table 8 Number of iterations for bicgstab2 preconditioned with multigrid as a function of the smallest cell width $h_{\min }$ (in m)

\begin{tabular}{rlrr}
\hline$h_{\min }$ & $\alpha$ & $10^{-6}$ & $10^{-8}$ \\
\hline 50 & 0.035 & 7 & 15 \\
20 & 0.059 & 30 & 75 \\
10 & 0.074 & 76 & 163 \\
5 & 0.089 & 157 & 409 \\
\hline
\end{tabular}

The largest ratio of cell widths between neighbouring cells is given by $1+\alpha$. Results are given for a drop of the residual by a factor of $10^{-6}$ and $10^{-8}$, respectively

Table 9 Parameters $k_{0}$ and $b$ found for the grid stretching based on the hyperbolic cosine function

\begin{tabular}{rllllll}
\hline$h_{\min }$ & $x: k_{0}$ & $b$ & $x: k_{0}$ & $b$ & $z: k_{0}$ & $b$ \\
\hline 50 & 63.0 & 0.035 & 63.0 & 0.035 & 67.5 & 0.027 \\
20 & 63.4 & 0.057 & 63.4 & 0.057 & 66.0 & 0.050 \\
10 & 63.5 & 0.071 & 63.5 & 0.071 & 65.5 & 0.065 \\
5 & 63.6 & 0.085 & 63.6 & 0.085 & 65.3 & 0.079 \\
\hline
\end{tabular}

cell width $h_{\min }$. The resulting parameters are listed in Table 9. The largest ratio between neighbouring cells is given by $1+\alpha$. The values of $\alpha$ can be found in Table 8 . Multigrid has been used as a preconditioner for bicgstab2. Again, we observe a performance degradation with increased grid stretching.

Cross-sections of the electric field components for $h_{\min }=20 \mathrm{~m}$ are displayed in Figs. 5, 6, and 7. The amplitude and phase of the electric and magnetic field components at the sea-bottom receivers are shown in Fig. 8, for the grid with a smallest cell width of $20 \mathrm{~m}$. The vertical component is not measured in practice.

\section{Discussion and conclusions}

A multigrid method for a finite-integration technique discretisation of the diffusive electromagnetic equations on tensor-product cartesian grids was applied to number of test problems. Textbook convergence rates were observed on equidistant grids. Convergence rates deteriorated on stretched grids, unless very mild stretching was used. 

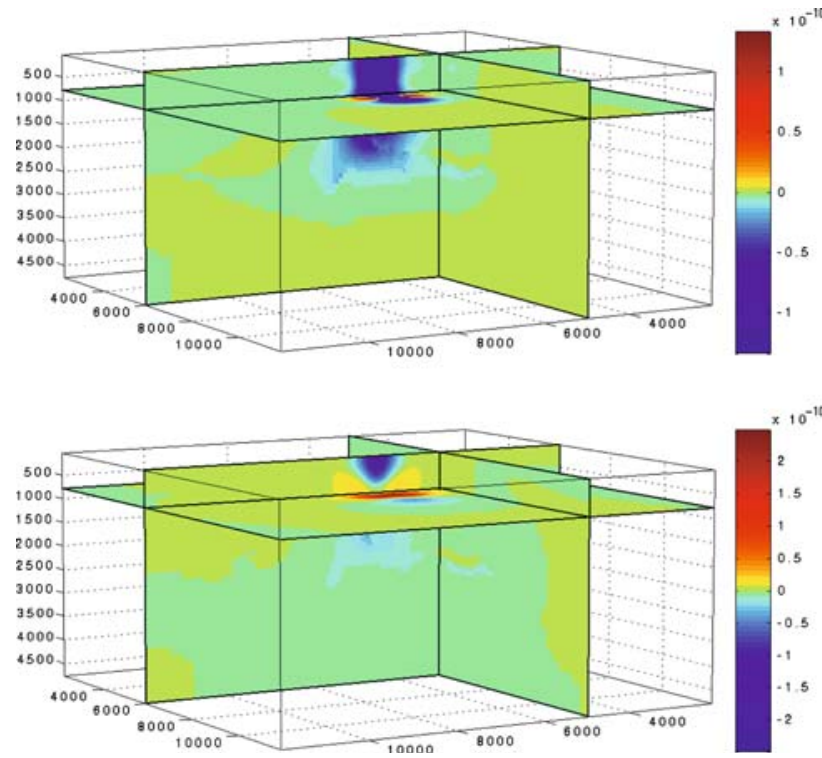

Fig. 5 Real and imaginary part of the electric field component $E_{1}$
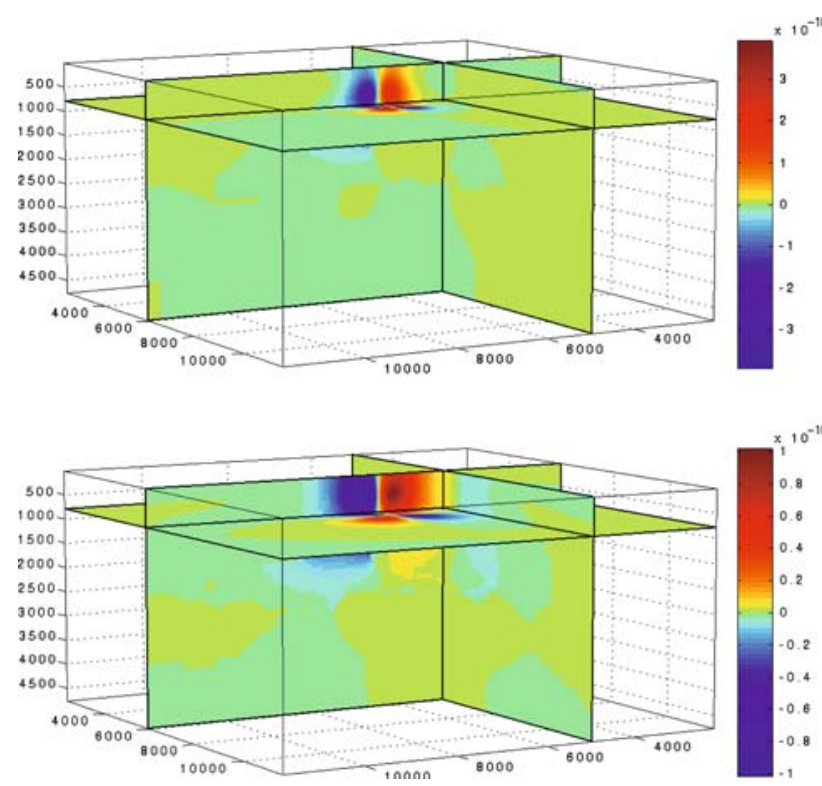

Fig. 6 Real and imaginary part of the electric field component $E_{2}$

The stretching introduces anisotropy in the discrete equations, which is well-known problem for multigrid methods. The are several potential remedies:

- A Krylov subspace method can be used to remove solution components that cause slow convergence. Here, it was found that bicgstab2 helped, but not enough.

- Line-relaxation, for instance symmetric line GaussSeidel, can be used to remove the effects of strong anisotropy. An example can be found in [24]. For 3D
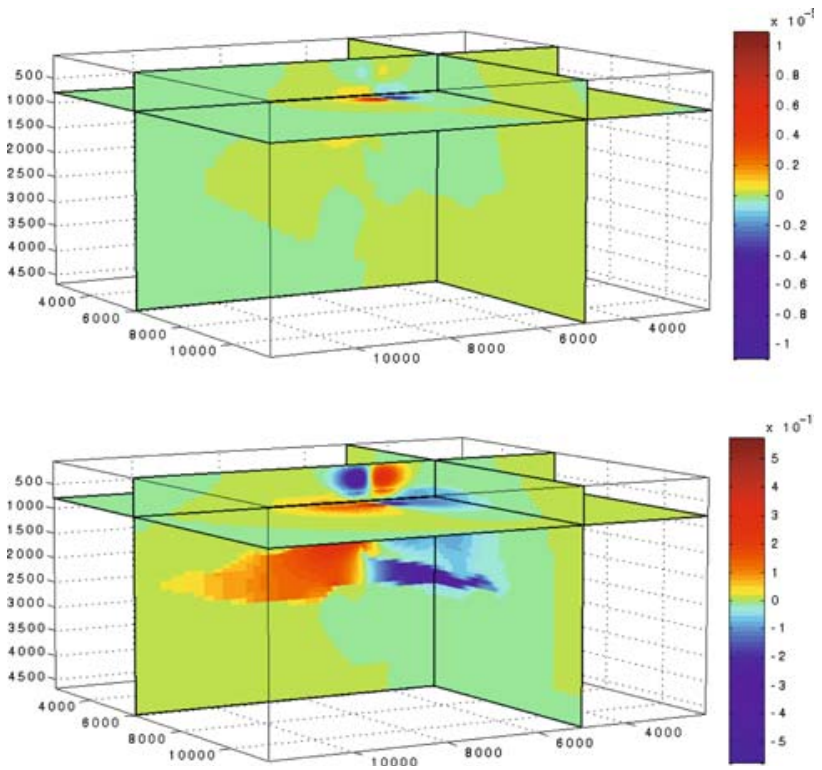

Fig. 7 Real and imaginary part of the electric field component $E_{3}$

problems, plane relaxation may be required, which is rather costly.

- Semi-coarsening can accomplish the same. In the standard approach, the grid is coarsened in only one coordinate at the time. A scheme that uses all direction simultaneously was introduced in [25]. The associated cost increase may be reduced by skipping some of the coarser grids.

- The adverse effects of strongly varying coefficients can be reduced by using operator-weighted restriction and prolongation operators [34].

- Another approach is to use a different way of defining coarser grid, for instance by not coarsening cells if they have large widths relative to the smallest cell [26], or by using coarser grids with nodes that do not coincide with the finer grid [14].

- An alternative is the computer-science solution of local grid refinement on equidistant grids. This should give very good convergence rates while still allowing for finer grids where necessary, at the expense of increased code complexity.

All of these methods will increase the cost of a multigrid iteration. It remains to be studied which is the most cost-effective one.

\section{Appendix: Grid stretching}

Here, we consider two types of grid stretching, based on a power-law and on the hyperbolic cosine function. 
Fig. 8 Electric and magnetic field at the receivers for a $128^{3}$ grid with a smallest grid spacing of $20 \mathrm{~m}$
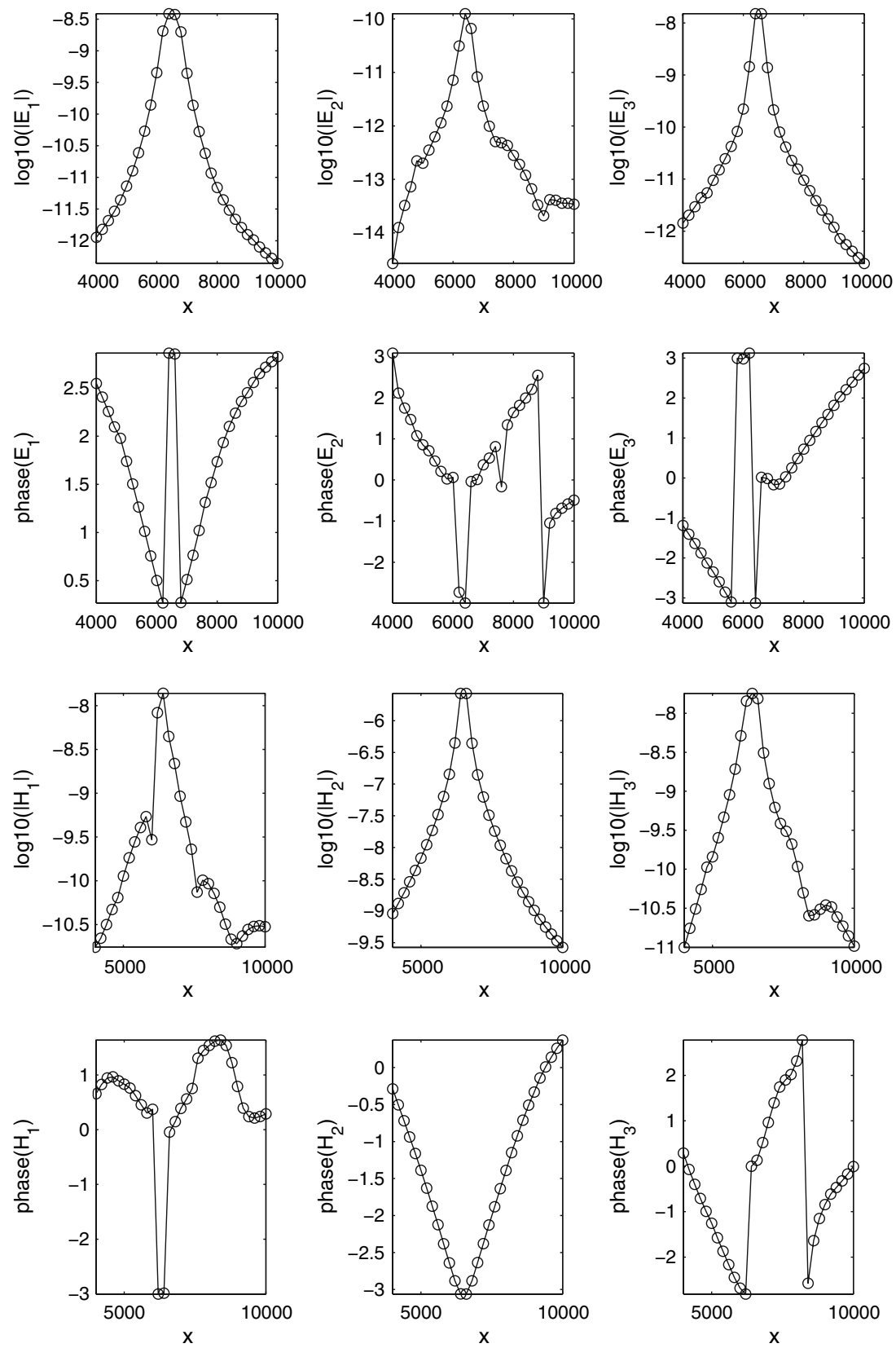

We start with former. The grid starts at $x=x_{\min }$ and ends at $x=x_{\max }$. The grid is stretched with respect to some reference point $x_{0}$ inside the domain. There are $n_{\mathrm{L}}$ cells to the left of $x_{0}$ and $n_{\mathrm{R}}$ to the right, with a total of $n=n_{\mathrm{L}}+n_{\mathrm{R}}$ cells. The grid spacings or cell widths are given by

$h_{k}=h a^{\left(n_{\mathrm{L}}-1-k\right)}, \quad$ for $k=0, \ldots, n_{\mathrm{L}}-1$,

$h_{k}=h a^{\left(k-n_{\mathrm{L}}\right)}, \quad$ for $k=n_{\mathrm{L}}, \ldots, n-1$.

Here $a=1+\alpha$. After summation, we have

$\frac{a^{n_{\mathrm{R}}}-1}{a-1}=\left(x_{\max }-x_{0}\right) / h, \quad \frac{a^{n_{\mathrm{L}}}-1}{a-1}=\left(x_{0}-x_{\min }\right) / h$.
To determine $n_{\mathrm{L}}$ and $n_{\mathrm{R}}=n-n_{\mathrm{L}}$, we determine the smallest integer $n_{\mathrm{L}}$ that minimises

$\left|\left(a^{\left(n-n_{\mathrm{L}}\right)}-1\right) /\left(a^{n_{\mathrm{L}}}-1\right)-r\right|$,

where

$r=\left(x_{\max }-x_{0}\right) /\left(x_{0}-x_{\min }\right)$.

Next, $h$ is determined such that $\sum_{k=0}^{n-1} h_{k}=x_{\max }-x_{\min }$.

For the hyperbolic cosine function, the grid spacing obeys $h_{k}=h_{r} \cosh \left(b\left(k-k_{0}+\frac{1}{2}\right)\right), k=0, \ldots, n-1$. For integer $k_{0}$, we have 


$$
\begin{aligned}
x_{0}-x_{\min } & =\sum_{k=0}^{k_{0}-1} h_{k}=\frac{1}{2} h_{r} \sinh \left(b k_{0}\right) / \sinh \left(\frac{1}{2} b\right), \\
x_{\max }-x_{0} & =\sum_{k=k_{0}}^{n-1} h_{k}=\frac{1}{2} h_{r} \sinh \left(b\left(n-k_{0}\right)\right) / \sinh \left(\frac{1}{2} b\right) .
\end{aligned}
$$

Using the same $r$ as before, we compute

$$
\begin{aligned}
k_{0}=\frac{1}{2} n+b^{-1} \sinh ^{-1}[ & (1-r) \sinh \left(\frac{1}{2} b n\right) \\
& \left./ \sqrt{1+r^{2}+2 r \cosh (b n)}\right],
\end{aligned}
$$

which generally is not an integer. This is ignored and the $h_{k}$ are rescaled by a constant factor such that $\sum_{k=0}^{n-1} h_{k}=$ $x_{\max }-x_{\min }$.

\section{References}

1. Aminzadeh, F., Brac, J., Kunz, T.: 3-D Salt and overthrust models. Society of Exploration Geophysicists, Tulsa (1997)

2. Amundsen, H.E.F., Johansen, S., Røsten, T.: A Sea Bed Logging (SBL) calibration survey over the Troll gas field. EAGE 66th Conference \& Exhibition, Paris, France, June 7-10, 2004

3. Arnold, D.N., Falk, R.S., Winther, R.: Multigrid in H(div) and H(curl). Numerische Mathematik 85, 197-217 (2000)

4. Aruliah, D.A., Ascher, U.M., Haber, E., Oldenburg, D.: A method for the forward modelling of 3D electromagnetic quasi-static problems. Math. Models Methods Appl. Sci. 11, 1-21 (2001)

5. Aruliah, D.A., Ascher, U.M.: Multigrid preconditioning for Krylov methods for time-harmonic Maxwell's equations in 3D. SIAM J. Sci. Comput. 24, 702-718 (2003)

6. Cheesman, S.J., Edwards, R.N., Chave, A.D.: On the theory of seafloor conductivity mapping using transient electromagnetic systems. Geophysics 52, 204-217 (1987)

7. Clemens, M., Weiland, T.: Discrete electromagnetism with the Finite Integration Technique. Prog. Electromagn. Res. (PIER) 32, 65-87 (2001)

8. Constable, S.C., Parker, R.L., Constable, C.G.: Occam's inversion: a practical algorithm for generating smooth models from EM sounding data. Geophysics 52, 289-300 (1987)

9. Cox, C.S., Constable, S.C., Chave, A.D., Webb, S.C.: Controlled source electromagnetic sounding of the oceanic lithosphere. Nature 320, 52-54 (1986)

10. Doll, H.G.: Introduction to induction logging and application to logging of wells drilled with oil base mud. J. Pet. Technol. 1, 148-162 (1949)

11. Edwards, R.N., Chave, A.D.: A transient electric dipoledipole method for mapping the conductivity of the seafloor. Geophysics 51, 984-987 (1986)

12. Eidesmo, T., Ellingsrud, S., Kong, F.N., Westerdahl, H., Johansen, S.: Method and apparatus for determining the nature of subterranean reservoirs, Patent application number WO 00/13046, filed August (1998)

13. Ellingsrud, S., Eidsmo, T., Johansen, S., Sinha, M.C., MacGregor, L.M., Constable, S.: Remote sensing of hydrocarbon layers by seabed logging (SBL): results from a cruise offshore Angola. Lead. Edge 21, 972-982 (2002)

14. Feigh, S., Clemens, M., Weiland, T.: Geometric Multigrid Method for Electro- and Magnetostatic Field Simulation Using the Conformal Finite Integration Technique. 2003
Copper Mountain Conference on Multigrid Methods, Copper Mountain, Colorado, (2003) (http://www.mgnet.org/ mgnet-cm2003.html)

15. George, A., Liu, J.: Computer Solution of Large Sparse Positive Definite Systems. Prentice-Hall, Englewood Cliffs (1981)

16. Gutknecht, H.H.: Variants of BiCGStab for matrices with complex spectrum. SIAM J. Sci. Stat. Comput. 14, 1020-1033 (1993)

17. Haber, E., Ascher, U.M.: Fast finite volume simulation of 3D electromagnetic problems with highly discontinuous coefficients. SIAM J. Sci. Stat. Comput. 22, 1943-1961 (2001)

18. Hiptmair, R.: Multigrid method for Maxwell's equations. SIAM J. Numer. Anal. 36, 204-225 (1998)

19. Horton, C.W.: On the use of electromagnetic waves in geophysical prospecting. Geophysics 11, 505-517 (1946)

20. MacGregor, L., Sinha, M.: Use of marine controlled-source electromagnetic sounding for sub-basalt exploration. Geophys. Prospect. 48, 1091-1106 (2000)

21. Meju, M.A., Gallardo, L.A., Mohamed, A.K.: Evidence for correlation of electrical resistivity and seismic velocity in heterogeneous near-surface materials. Geophys. Res. Lett. 30(7), 1373-1376 (2003)

22. Monk, P., Süli, E.: A convergence analysis of Yee's scheme on nonuniform grids. SIAM J. Numer. Anal. 31, 393-412 (1994)

23. Moran, J.H., Kunz, K.S.: Basic theory of induction logging and application to the study of two-coil sondes. Geophysics 27, 829-858 (1962)

24. Mulder, W.A.: Computation of the quasi-steady gas flow in a spiral galaxy by means of a multigrid method. Astron. Astrophys. 156, 354-380 (1986)

25. Mulder, W.A.: A new multigrid approach to convection problems. J. Comput. Phys. 83, 303-323 (1989)

26. Pöplau, G., van Rienen, U.: Multigrid solvers for Poisson's equation in computational electromagnetics. In: van Rienen, U., Günther, M., Hecht, D. (eds.) Scientific Computing in Electrical Engineering, Lecture Notes in Computational Science and Engineering, vol. 18, pp. 169-176. Springer, Berlin (2001)

27. van Rienen, U.:Frequency domain analysis of waveguides and resonators with FIT on non-orthogonal triangular grids. Prog. Electromagn. Res. (PIER) 32, 357-381 (2001)

28. Smith, J.T.: Conservative modeling of 3-D electromagnetic fields. Part II: Biconjugate gradient solver and an accelerator. Geophysics 61, 1319-1324 (1996)

29. Statham, L.: Electric earth transients in geophysical prospecting. Geophysics 1, 271-277 (1936)

30. Strack, K.M.: Exploration with Deep Transient Electromagnetics. Elsevier, Amsterdam (1992)

31. van der Vorst, H.A.: BI-CGSTAB: a fast and smoothly converging variant of bi-CG for the solution of nonsymmetric linear systems. SIAM J. Sci. Stat. Comput. 13, 631-644 (1992)

32. Ward, S.H., Hohmann, G.W.: Electromagnetic theory for geophysical applications. In: Nabighian, M.N. (ed.) Electromagnetic Methods in Applied Geophysics-Theory, vol. 1, pp. 131-311. Society of Exploration Geophysicists, Tulsa (1987)

33. Weiland, T.: A discretization method for the solution of Maxwell's equations for six-components fields. Electron. Commun. 31, 116-120 (1977)

34. Wesseling, P.: An Introduction to Multigrid Methods. Wiley, New York (1992)

35. Yee, K.: Numerical solution of initial boundary value problems involving Maxwell's equations in isotropic media. IEEE Trans. Antennas Propag. 16, 302-307 (1966)

36. Young, P.D., Cox, C.S.: Electromagnetic active source sounding near the East Pacific Rise. Geophys. Res. Lett. 8, 10431046 (1981) 